\title{
MONOCLONAL ANTIBODIES RECOGNISING SIALYL-TN: PRODUCTION AND APPLICATION TO IMMUNOCHEMISTRY
}

\author{
PETER L. DEVINE*, GEOFFREY W. BIRRELL*, RACHEL J. QUIN* AND PAUL W. SHIELD ${ }^{\dagger}$. \\ *Department of Obstetrics and Gynaecology, The University of Queensland, Australia \\ ${ }^{\dagger}$ Department of Cytology, Royal Brisbane Hospital, Queensland, Australia
}

\begin{abstract}
SUMMARY
In order to develop reagents that can detect the exposed sialyl-Tn antigen (NeuAc $\alpha 2,6 \mathrm{GalNA} \alpha \alpha 1-\mathrm{O}-\mathrm{Ser} / \mathrm{Thr}$ ) on tumour-associated mucins, we have prepared monoclonal antibodies (mabs 3C2 and 3D1, both IgM) against ovine submaxillary mucin (OSM; >98\% of glycans as sialyl-Tn). These mabs showed strong reactivity with OSM and bovine submaxillary mucin (BSM; $50 \%$ of glycans as sialyl-Tn) but did not react with desialylated OSM or BSM. Sialic acid at $1 \mathrm{mg} / \mathrm{ml}$ did not significantly inhibit mab binding to OSM, suggesting that the linkage to GalNAc may be important for mab binding. 3C2 and 3D1 also showed similar reactivity to sialyl-Tn reactive mab B72.3, and detected B72.3 captured OSM in a sandwich ELISA. In Western blotting of mucus from a patient with a mucinous ovarian tumour, the mabs reacted with high molecular weight ( $>200 \mathrm{kDa}$ ) species. In immunohistochemistry, these mabs showed strong reactivity with most cancers of the colon, lung, and stomach, and also some tumours of the ovary and breast. There was only limited reactivity in normal tissue from these sites. The antibodies should be useful reagents for the detection of the sialyl-Tn antigen in human cancers.
\end{abstract}

KEYWORDS Sialyl-Tn Mucin Monoclonal antibody.

\section{INTRODUCTION}

Mucins are high molecular weight glycoproteins containing up to $80 \%$ by weight $\mathrm{O}$-linked carbohydrate (Devine and McKenzie,1992). They are located at the epithelial surface or secreted to form mucus. Apart from increased mucin synthesis and secretion, blocked or neosynthesis of O-linked carbohydrate chains can also occur in tumours, resulting in the exposure of novel epitopes such as the sialyl-Tn antigen (NeuAc $\alpha 2,6 \mathrm{GalNAc})$ (Hakomori,1989). Expression of this antigen in tumours or serum has been associated with poor prognosis in patients with gastric (Maeda et al.,1994; Ma et al.,1993; Takahashi et al.,1993), colonic (Itzkowitz et al.,1990), and ovarian (Kobayashi et al.,1992) carcinomas, and serum tests for this antigen have also been shown to be useful in the clinical management of patients with these cancers (Motoo et al.,1991; Kobayashi et al.,1991; Inoue et al.,1990; Kyuko et al.,1992). In this paper we describe the production of sialyl-Tn-reactive monoclonal antibodies by immunising with ovine submaxillary mucin.

Correspondence to: Dr. Peter Devine, Dept. of Obstetrics and Gynaecology, Clinical Sciences Bldg., Royal Brisbane Hospital, Herston, Qld 4029, Australia. Tel. 07-3655202; Fax 07-3655203. 


\section{MATERIALS AND METHODS}

\section{Monoclonal Antibody Production}

A BALB/c female mouse ( 8 weeks old) was injected by sc and im routes with OSM (Auspep, Parkville, Australia) emulsified in Freund's Complete Adjuvant ( $1 \mathrm{mg} / \mathrm{mL}$ ). Further injections were given one and two months later, except the mucin was emulsified in Freund's Incomplete Adjuvant (FIA) and injected by ip and im routes. After a further month, the mouse was injected iv with OSM in PBS and ip with OSM in FIA. The spleen cells were fused with Ag8 cells four days later.

Hybridomas were screened by ELISA on OSM and desialylated OSM as a negative control. Two mabs, 3C2 and 3D1, showed specificity for OSM, and these were cloned by limiting dilution. The subclass of the mab was determined by dual-determinant ELISA using subclass-specific antibodies to capture mab and anti-mouse Ig-peroxidase (Silenus, Australia) to detect bound mab.

\section{Glycoproteins and Carbohydrates}

Glycoproteins used were ovine submaxillary mucin (OSM) (Auspep, Parkville, Australia), bovine fetuin and asialofetuin (Sigma Chem. Co., MO, USA), bovine submaxillary mucin (BSM) (Sigma Chem. Co., MO, USA), porcine stomach mucin (PSM) (Sigma Chem. Co., MO, USA), bovine serum albumen (BSA) (Sigma Chemical Co., MO, USA), and human transferrin (Boehringer-Mannheim, Germany). Native and TFMSA-treated cystic fibrosis (CF) mucin was kindly donated by Dr. Goverdan Sachdev (Desai et al.,1991) while native and TFMSA-treated asthma mucin was kindly provided by Dr. David Thornton (Thornton et al.,1994). Asialomucins were prepared by incubation of $2 \mathrm{mg} / \mathrm{ml}$ mucin in PBS with an equal volume of $1 \mathrm{U} / \mathrm{ml}$ Vibrio cholerae neuraminidase (Hoechst-Behring, Marburg, Germany) for 3 hours at $37^{\circ} \mathrm{C}$. All monosaccharides used in this study were purchased from the Sigma Chemical Co., MO, USA.

\section{ELISA}

All assays were performed in duplicate, with the $\% \mathrm{CV}$ of duplicates being $<10 \%$ in all cases. Concentrations of reagents used are given in figure legends.

Solid-Phase ELISA: Antigens $(50 \mu \mathrm{l} /$ well) were coated onto a Falcon flexible assay plate (Becton Dickinson, U.S.A.) by incubating overnight at $4^{\circ} \mathrm{C}$ in $0.1 \mathrm{M}$ carbonate buffer $\mathrm{pH}$ 9.6. Plates were blocked for 1 hour at room temperature (RT) with $75 \mu \mathrm{l} /$ well $2 \%(\mathrm{w} / \mathrm{v}) \mathrm{BSA}$ in PBS, and incubated overnight with mab in phosphate buffered saline pH 7.2 containing $0.05 \%$ Tween-20 (PBS-Tween). Bound mab was detected using anti-mouse Ig-peroxidase (Silenus, Australia) and ABTS substrate (Devine et al.,1990). Plates were washed three times with PBS-Tween between each incubation, and checkerboard titrations of antigen and mab were performed (Devine et al.,1991).

Inhibition ELISA: Optimal concentrations of antigens and mabs were determined by checkerboard ELISA, and inhibition ELISA was performed (Devine et al.,1991). Briefly, mabs at twice the concentration for $50 \%$ binding were incubated for three hours at RT with an equal volume of glycoprotein solution in PBS-Tween. "No inhibition" (mab plus PBS-Tween) and "full inhibition" (PBS-Tween, no mab) incubations were also performed. Subsequently, $50 \mathrm{ul}$ was transferred to a OSM-coated plate and the assay was completed as above. Inhibition was calculated as described (Layton et al.,1987). 
Two-Site ELISA: Microtitre plates were coated with B72.3 (Dako, CA, USA) by an overnight incubation in carbonate $\mathrm{pH} 9.6$ at RT. Plates were blocked with BSA as described above, and glycoproteins in PBS-Tween were added for 3 hours at RT. After washing the plates, 3A2 or 3D1 (both IgM) were added for 1 hour at RT, followed by anti-mouse IgM-specific peroxidase (Silenus, Parkville, Australia) at 1/1000 for 1 hour at RT. ABTS was used as substrate.

\section{Western Blotting}

Proteins were separated on 3-15\% non-reducing SDS-polyacrylamide gels, and Western blotting was performed as described (Devine et al.,1990). Mucinous tumour from a patient with ovarian cancer was solublised in a buffer containing $1 \%$ SDS and 5\% mercaptoethanol as described (Devine et al.,1990).

\section{Immunohistochemistry}

Formalin fixed, paraffin embedded specimens were selected from files of the Anatomical Pathology Departments, The Prince Charles Hospital and Royal Brisbane Hospital. For each case, representative blocks were selected and sections $5 \mu \mathrm{m}$ thick were cut and mounted on glass slides for immunochemical staining. Selected specimens included carcinomas of the colon $(n=11)$, stomach $(n=10)$, lung $(n=37)$, ovary $(n=16)$, and breast $(n=9)$. Normal tissue from the edge of the resection margins was also present in many of these specimens. In addition, a further 5 specimens from each site containing only normal epithelium were stained with $3 \mathrm{C} 2$ and 3D1.

Specimens were stained with a standard streptavidin-biotin peroxidase method with DAB as the chromogen (Shield et al.,1994). Following dewaxing, rehydration and quenching of endogenous peroxidase with $4 \%$ hydrogen peroxide in $0.1 \%$ sodium azide, the sections were sequentially incubated with 3C2 and 3D1 (1:300) for 1 hour, biotinylated rabbit anti-mouse sera (Dako, Carpenteria, CA) (1:500) for 30 mins and streptavidin-biotin (Zymed, South San Francisco, CA) (1:4000) for 30 mins. Sections of normal colon were stained with each batch as positive controls. Negative controls were performed by substituting either $5 \%$ normal rabbit serum or FM1 (Medical Innovations, Labrador, Queensland) (diluted 1:300), a mouse MAb which is non-reactive with human tissue, for 3C2 and 3D1. Staining patterns were evaluated with regard to the intensity, distribution and localisation of staining in tissues and cells. In tumour tissue, the distribution of staining was recorded as being either absent, or present in less than $25 \%, 26-50 \%, 51-75 \%$, or more than $76 \%$ of tumour cells. Cellular localisation of staining was categorised as membranous, cytoplasmic or both.

\section{RESULTS}

\section{Production of sialyl-Tn reactive monoclonal antibodies}

Monoclonal antibodies 3C2 and 3D1 (both IgM) were chosen after the fusion, as these reacted with OSM but not asialo-OSM. Control mab FM1 was negative in all cases. Checkerboard titration showed that the reactivity of these mabs with OSM was concentration dependent and similar to that observed with commercially-available sialyl-Tn-reactive mab B72.3 (Figure 1). 


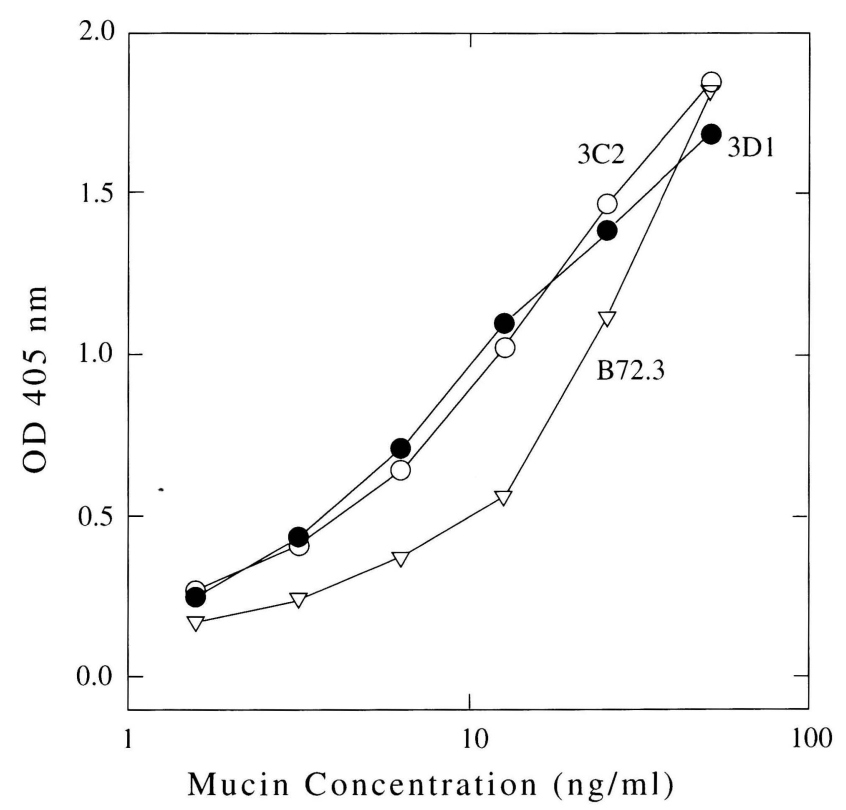

Figure 1. Reactivity of mabs 3C2 (O), 3D1 $(\bullet)$, and B72.3 $(\nabla)$ with ovine submaxillary mucin $(\mathrm{OSM})$ in solid phase ELISA: titration of OSM. 3C2 and 3D1 hybridoma culture supernatants were used at 1/64 in PBS-Tween, while B72.3 (supplied as ascites diluted in PBS) was used at 1/6400 in PBS-Tween.

\section{Reactivity of Antibodies in ELISA}

In solid phase ELISA, 3C2 (Figure 2) and 3D1 (not shown) reacted with OSM, bovine submaxillary mucin (BSM), and cystic fibrosis mucin (CFM), but not with porcine stomach mucin (PSM). Neuraminidase treated OSM or BSM, TFMSA treated CFM, human asthma mucin, fetuin, asialofetuin, transferrin, and BSA showed no reactivity with mabs (not shown). Control antibody FM1 was negative in all cases.

In inhibition ELISA, 3C2 and 3D1 binding to OSM was inhibited strongly by OSM, moderately by BSM, and not by PSM (Figure 3). The concentrations of OSM required for $50 \%$ inhibition of mabs were $9 \mathrm{ng} / \mathrm{ml}(3 \mathrm{C} 2), 10 \mathrm{ng} / \mathrm{ml}(3 \mathrm{D} 1)$, and $35 \mathrm{ng} / \mathrm{ml}(\mathrm{B} 72.3)$. Higher concentrations of BSM were required for $50 \%$ inhibition of mabs. These were 0.5 $\mu \mathrm{g} / \mathrm{ml}(3 \mathrm{C} 2), 0.75 \mu \mathrm{g} / \mathrm{ml}(3 \mathrm{D} 1)$, and $3.0 \mu \mathrm{g} / \mathrm{ml}(\mathrm{B} 72.3)$.

In two-site ELISA, 3C2 and 3D1 both reacted strongly with OSM captured by B72.3, while control mab FM1 was negative (Figure 4).

\section{Western Blotting}

Following 3-15\% PAGE and Western transfer of mucus from a patient with an ovarian carcinoma, a number of high molecular weight bands $(>200 \mathrm{kDa})$ were detected with $3 \mathrm{C} 2$ and 3D1 (Figure 5). Positive control mab 4E7 (anti-CEA) reacted with a series of lower molecular weight bands in this preparation (lane c), while negative control mab FM1 gave no reactivity (lane d). 


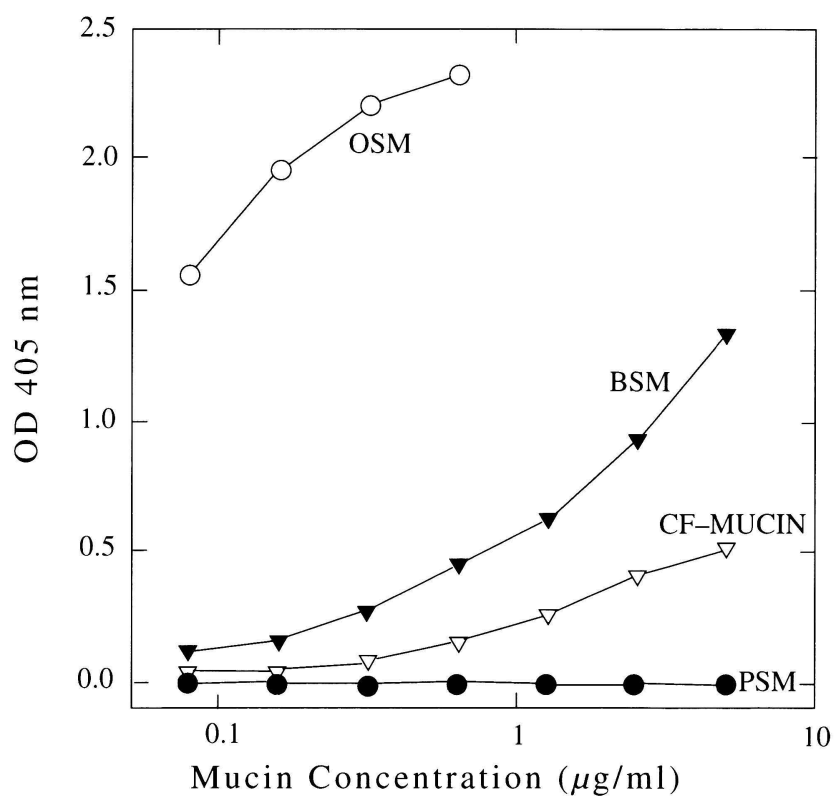

Figure 2. Reactivity of 3C2 with OSM ( $\bigcirc$ ), BSM ( $\nabla$ ), PSM ( $\bigcirc$ ), and CF-mucin $(\nabla)$ by solid phase ELISA: titration of mucins. 3C2 hybridoma culture supernatant was used at $1 / 15$ in PBS-Tween. Neuraminidase-treated OSM and BSM, and TFMSA-treated CF-mucin were negative (not shown).

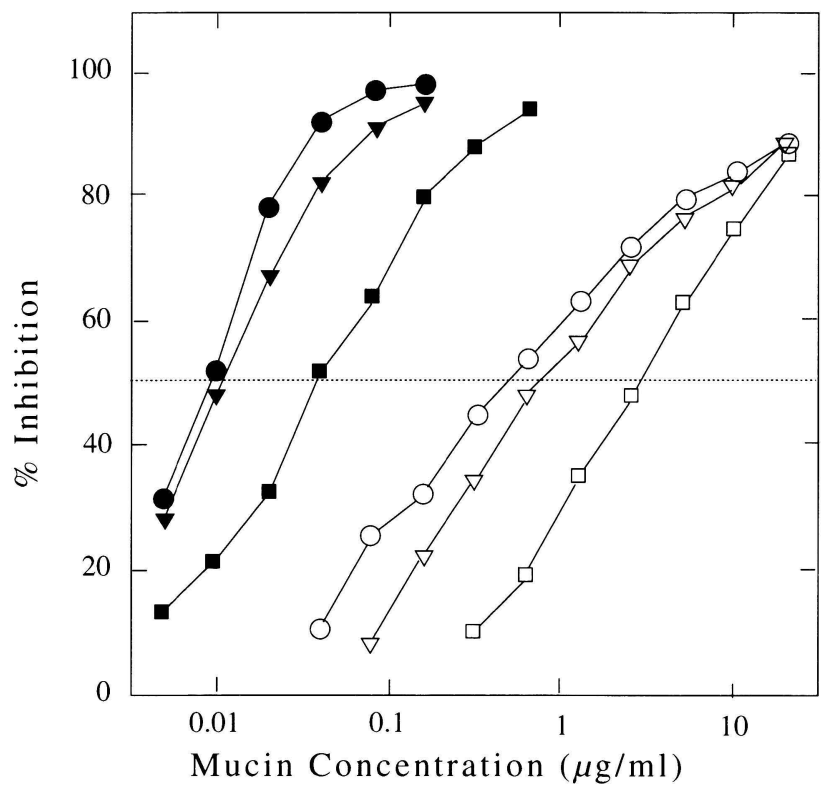

Figure 3. Inhibition of 3C2 (circle), 3D1 (triangle), and B72.3 (square) binding to an OSM-coated assay plate $(0.05 \mu \mathrm{g} / \mathrm{ml})$ by OSM (closed symbols) and BSM (open symbols): titration of inhibitors. 3C2 and 3D1 hybridoma culture supernatants were used at 1/75 in PBS-Tween (final dilution 1/150), while B72.3 (supplied as ascites dilutued in PBS) was used at 1/12500 in PBS-Tween (final dilution 1/25000). PSM (not shown) showed no inhibiton of these mabs at the concentrations shown. 


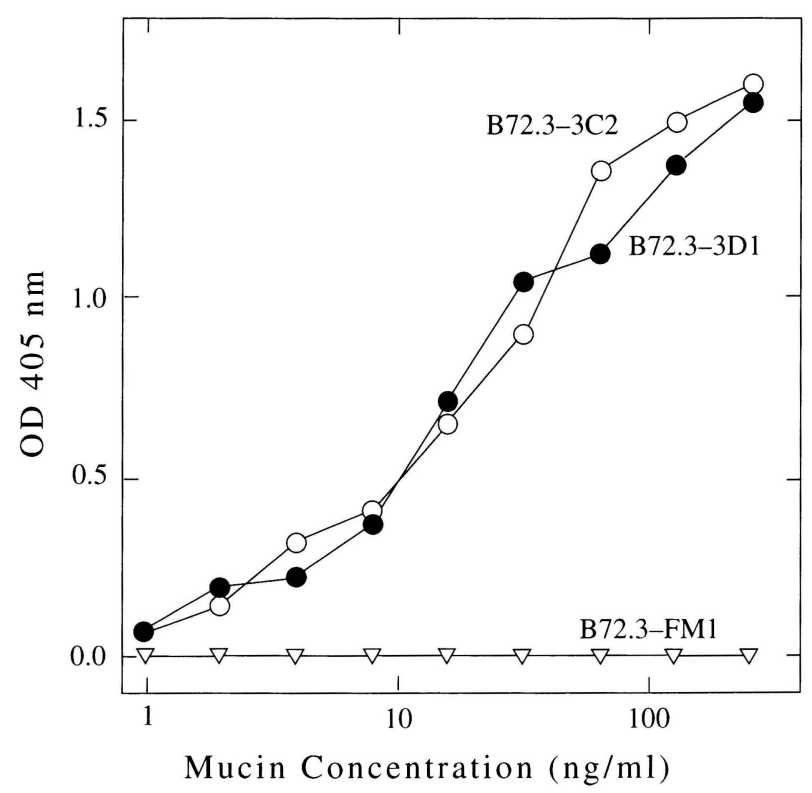

Figure 4. Reactivity of OSM in sandwich ELISA using B72.3 (IgG1) to capture mucin and 3C2, 3D1, or FM1 (IgM) to detect bound mucin: titration of OSM. B72.3 (supplied as ascites diluted in PBS) was coated onto the assay plate at $1 / 200$, while detection mabs (hybridoma culture supernatants) were used at $1 / 2$ in PBS-Tween.
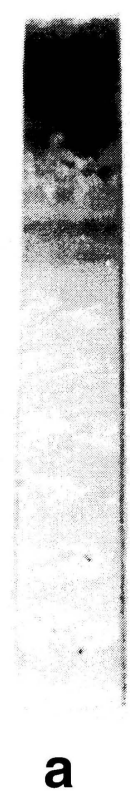
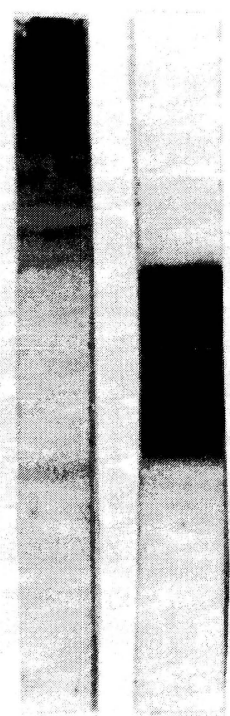

b

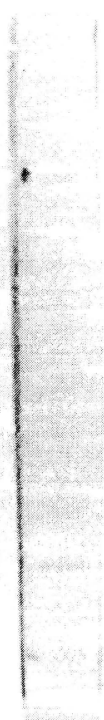

C

d

Figure 5. Reactivity of 3C2, 3D1, and control mabs FM1 and 4E7 with an extract of a mucinous ovarian tumour by Western blotting. Antibodies shown are 3C2 (a), 3D1 (b), positive control 4E7 (c), and negative control FM1 (d). All mabs were used as ascites diluted 1/1000 with PBS-Tween containing 5\% donor calf serum. 
Reactivity of antibodies with non-malignant tissue by immunoperoxidase staining

Both antibodies showed a similar pattern of reactivity with benign tissue from all sites examined. In benign gastric epithelium, 3C2 and 3D1 staining was limited to secretory vacuoles of goblet cells and secretions present in the lumen of glands (Figure 6a). Colonic epithelium was uniformly negative in most cases, however three cases contained occasional goblet cells positive with both antibodies. In addition, a single case demonstrated positive staining with $3 \mathrm{C} 2$ in superficial epithelial cells of the colon. In these cells staining was localised in the perinuclear cytoplasm. In lung tissue, staining was present in occasional bronchial epithelial cells $(<20 \%)$ and a few cells in some mucus glands. Bronchial epithelial cells showed granular perinuclear staining (Figure 6b). No staining was observed in normal breast epithelium or in 5 cases of benign ovarian tumours.

\section{Reactivity of antibodies with malignant tumours by immunoperoxidase staining}

In general, the two antibodies showed similar reactivity with all tumour types tested (Table 1). Both antibodies labelled a majority of gastric, colon and lung adenocarcinomas (Figures 6c and 6d). Breast carcinomas and ovarian carcinomas were less frequently positive. Three tumours were negative with $3 \mathrm{C} 2$ but positive with $3 \mathrm{D} 1$ (two lung adenocarcinomas and one mucinous ovarian tumour), while five tumours were positive for 3C2 and negative for 3D1 (two squamous cell carcinomas and one adenocarcinoma of the lung, one colonic carcinoma and one mucinous ovarian tumour). Staining with 3C2 and 3D1 was often focal within tumour masses. In malignant cells staining was usually granular and cytoplasmic, as was noted in normal cells. However in most malignant cases, cellular staining was distributed throughout the cytoplasm, rather than compartmentalised. In some cases strong membrane staining of malignant cells was also present.

\section{DISCUSSION}

The reactivity of 3C2 and 3D1 with sialyl-Tn (NeuAc $\alpha 2,6$ GalNAc $\alpha 1-O-S e r / T h r)$ was confirmed by ELISA, where these mabs reacted with OSM (more than $98 \%$ of glycans as sialyl-Tn) (Kurosaka et al.,1988) and BSM (53\% of glycans as sialyl Tn) (Tsuji and Osawa,1986), but not with desialylated OSM or BSM. These mabs showed no reactivity with PSM (22-47\% as sialyl-Tn) in solid phase ELISA and only very weak reactivity with PSM in inhibition ELISA since sialic acid is in the form of N-glycolyl neuraminic acid rather than the acetyl derivative (Kurosaka et al.,1988). Surprisingly, NeuAc at $1 \mathrm{mg} / \mathrm{ml}$ showed no inhibition of 3C2 and 3D1 binding to OSM, which may indicate the importance of the linkage to GalNAc (not shown). Furthermore, these mabs showed similar reactivity to another mab with reported specificity for sialyl-Tn (B72.3) (Kurosaka et $a l ., 1988$ ), and reacted with B72.3 captured mucin in two-site ELISA. As expected, a number of high molecular weight mucin-like species were detected in Western blotting.

The observations from immunohistochemical staining of both normal and malignant tissue are consistent with 3C2 and 3D1 recognising sialyl-Tn. The tissue and cellular distribution of staining in normal gastrointestinal and respiratory tissue with 3C2 and 3D1 was similar to that described for other sialyl-Tn reactive Mabs, B72.3 and TKH2 (Yonezawa et al.,1992; Loy and Nashelsky, 1993). The epitope has a restricted expression in these sites but was not found in normal breast tissue or in 5 cases of benign ovarian tumour. 

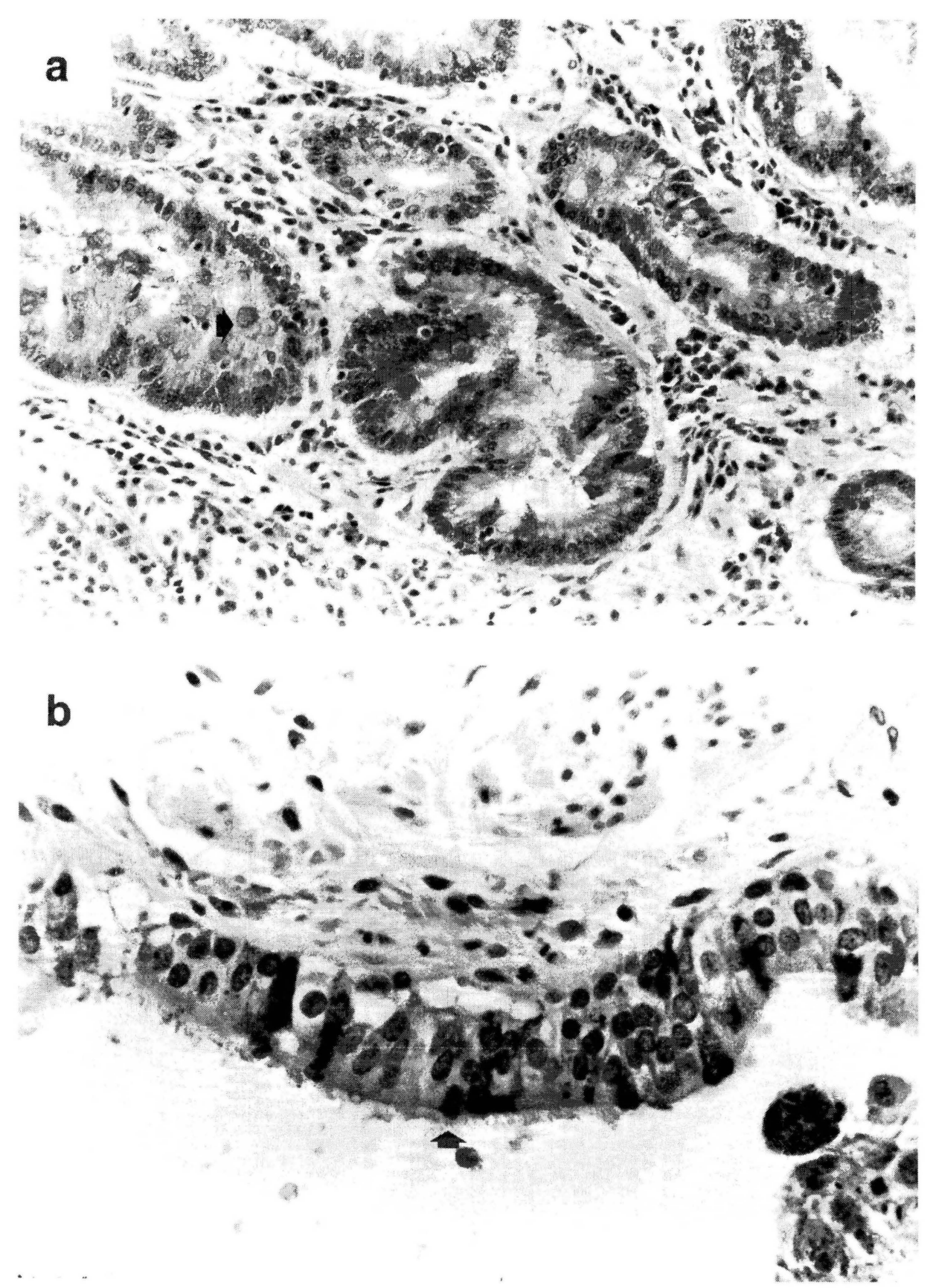


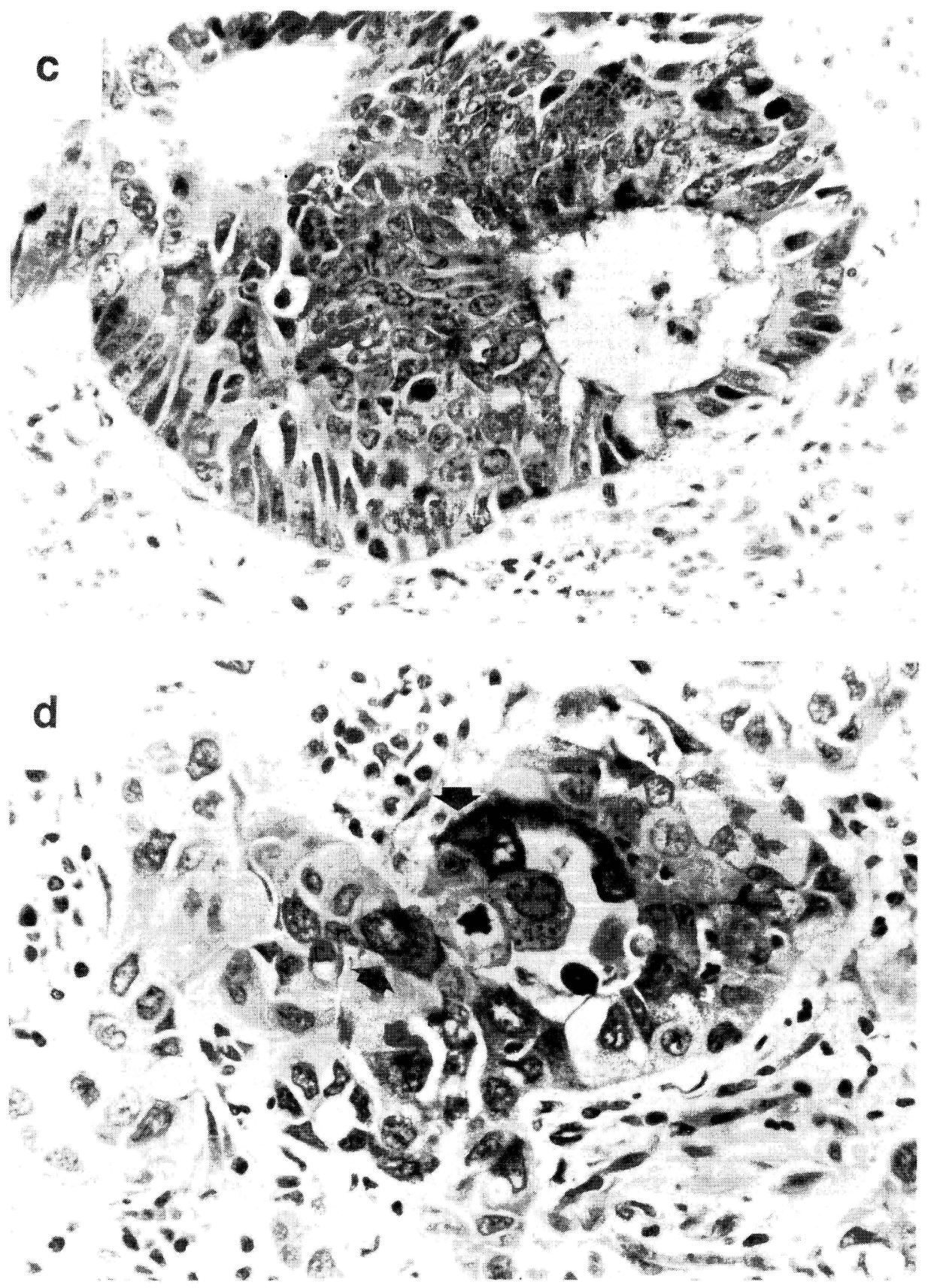

Figure 6. Immunohistochemical reactivity of mabs with (a) normal gastric tissue stained with 3D1 (x200). Epithelial cells show cytoplasmic positivity, particularly in secretory vacuoles (arrows). Luminal secretions are also positive; (b) normal bronchial epithelium stained with 3C2 (x400). Intense granular perinuclear staining is present (arrows); (c) colonic adenocarcinoma cells stained with 3 C2 (x200). The cells have granular staining distributed throughout the cytoplasm; and (d) squamous cell carcinoma of the lung stained with 3D1 (x400). Strong focal cytoplasmic staining is present (arrows). All slides were stained with streptavidin-biotin peroxidase, with DAB chromogen and haematoxylin (17). 
Table 1. Immunohistochemical reactivity of 3C2 and 3D1 with carcinomas.

\begin{tabular}{|c|c|c|c|c|c|}
\hline \multirow[t]{2}{*}{ Tumour (n) } & \multicolumn{5}{|c|}{ Percentage of Tumour Cells Stained } \\
\hline & $0 \%$ & $1-25 \%$ & $26-50 \%$ & $51-75 \%$ & $>76 \%$ \\
\hline & \multicolumn{5}{|c|}{ No. Cases Reacting with $3 \mathrm{C} 2$} \\
\hline Gastric (10) & 3 & 4 & 1 & 2 & 0 \\
\hline Colon (11) & 2 & 2 & 0 & 5 & 2 \\
\hline Lung SCC (16) & 6 & 8 & 1 & 1 & 0 \\
\hline Lung Adeno (21) & 7 & 10 & 3 & 0 & 1 \\
\hline Ovary Muc (7) & 4 & 1 & 1 & 0 & 1 \\
\hline Ovary non-Muc (9) & 6 & 3 & 0 & 0 & 0 \\
\hline Breast (9) & 7 & 2 & 0 & 0 & 0 \\
\hline
\end{tabular}

No. Cases Reacting with 3D1

\begin{tabular}{llllll} 
Gastric (10) & 3 & 4 & 2 & 1 & 0 \\
Colon (11) & 3 & 1 & 3 & 3 & 1 \\
Lung SCC (16) & 8 & 7 & 0 & 1 & 0 \\
Lung Adeno (21) & 7 & 9 & 4 & 0 & 1 \\
Ovary Muc (7) & 4 & 2 & 0 & 0 & 1 \\
Ovary non-Muc (9) & 6 & 3 & 0 & 0 & 0 \\
Breast (9) & 7 & 2 & 0 & 0 & 0 \\
\hline
\end{tabular}

*Abbreviations used:

Ovary Muc: mucinous ovarian tumours; Ovary non-Muc: non-mucinous ovarian tumours; Adeno: adenocarcinoma; SCC: squamous cell carcinoma.

By contrast, staining with $3 \mathrm{C} 2$ and 3D1 was positive in a majority of carcinomas from the stomach, colon and lung, and in some breast and ovarian tumours. Immunohistochemical studies with B72.3 and TKH2 have detected sialyl-Tn in most adenocarcinomas from the lung, stomach, colon, pancreas, bile ducts, ovary, endometrium and prostate (Yonezawa et al.,1992; Loy and Nashelsky, 1993; David et al.,1992; Dahiya et al.,1992; Itzkowitz et al.,1989; Ryuko et al.,1993; Inoue et al.,1991). The epitope is also expressed in breast carcinomas, although in a smaller proportion (Loy and Nashelsky, 1993) and in some normal and neoplastic cells of the oral mucosa (Byrne et al.,1991). Sialyl-Tn is rarely expressed by hepatocellular carcinomas or mesotheliomas, and immunohistochemical staining for the antigen is therefore useful in resolving diagnostic problems involving these tumours in both histology and cytology specimens (Shield et al.,1994; Loy and Nashelsky, 1993; Sheibani et al.,1992; Ordonez et al.,1989; Nance and Silverman, 1991).

Increased immunohistochemical expression of sialyl-Tn by most colonic, gastric, and lung tumours, and by some ovarian and breast cancers demonstrates accumulation of this epitope in tumours after blocked glycan synthesis. This is consistent with the pattern of intracellular staining observed with $3 \mathrm{C} 2$ and $3 \mathrm{D} 1$, and also reported for $\mathrm{B} 72.3$ 
(Yonezawa et al.,1992, Loy and Nashelsky,1993). Immunohistochemical staining of tumour cells was generally more intense than that observed in normal cells. In addition, although staining in normal cells was always localised in perinuclear (golgi) regions or in secretory vacuoles, in malignant cells the staining was frequently distributed throughout the cell, and in some cases more intensely on the cell membrane. As has been noted with B72.3 (Sheibani et al.,1992), staining with both 3C2 and 3D1 was often focally distributed in tumours.

\section{ACKNOWLEDGEMENTS}

The authors wish to acknowledge the assistance of Mike McGuckin, Tony Dare, and Michael Walsh for cutting paraffin embedded tissue sections. Dr Devine and Ms Quin are employed on grants from the Queensland Cancer Fund and the University of Queensland Cancer Research Fund respectively.

\section{REFERENCES}

Byrne, M., Reibel, J., Mandel, U., Dabelsteen, E. (1991). Expression of mucin type carbohydrates may supplement histological diagnosis in oral premalignant lesions. J. Oral Pathol. Med., 20, 120-25.

Dahiya, R., Itzkowitz, S.H., Byrd, J.C., Kim, Y.S. (1992). Mucin oligosaccharide biosynthesis in human colonic cancerous tissues and cell lines. Cancer, 70, 1467-76.

David, L., Nesland, J.M., Clausen, H., Carneiro, F., Sobrinho-Simoes, M. (1992). Simple mucin-type carbohydrate antigens (Tn, Sialosyl-Tn and T) in gastric mucosa, carcinomas and metastases. APMIS, 100, 162-72.

Desai, V.C., Naziruddin, B., Graves, D.C., de la Rocha, S.R., Sachdev, G.P. (1991). Production and characterisation of monoclonal antibodies to purified deglycosylated cystic fibrosis respiratory mucin: evidence for the presence of four immunologically distinct epitopes. Hybridoma, 10, 285-96.

Devine, P.L., Warren, J.A., Clark, B.A., Layton, G.T., Ward, B.G., Macdonald, B., Xing, P.X., McKenzie, I.F.C. (1990). The complexity of cancer-associated epitope expression on antigens produced by ovarian tumour cells. J. Tumor Marker Oncol., 5, 321-39.

Devine, P.L., Clark, B.A., Birrell, G.W., Layton, G.T., Ward, B.G., McKenzie, I.F.C. (1991). The breast tumor-associated epitope defined by monoclonal antibody $3 \mathrm{E} 1.2$ is an O-linked mucin carbohydrate containing N-glycolyl neuraminic acid. Cancer Res., 51, 5826-36.

Devine, P.L., McKenzie, I.F.C. (1992). Mucins: structure, functions, and relationships with malignancy. BioEssays, 14, 619-25.

Hakomori, S. (1989). Aberrant glycosylation in tumors and tumor-associated carbohydrate antigens. Adv. Cancer Res., 52, 257-331.

Inoue, M., Ogawa, H., Nakanishi, K., Tanizawa, O., Karino, K., Endo, J. (1990). Clinical value of sialyl Tn antigen in patients with gynecologic tumours. Obstet. Gynecol., 75, 1032-36.

Inoue, M., Ogawa, H., Tanizawa, O., Kobayashi, Y., Tsujimoto, M., Tsujimura, T. (1991). Immunodetection of sialyl-Tn antigen in normal, hyperplastic and cancerous tissues of the uterine endometrium. Virchow's Arch. A Pathol. Anat. Histopathol., 418, 157-62.

Itzkowitz, S.H., Yuan, M., Montgomery, C.K., Kjeldsen, T., Takahashi, H.K., Bigbee, W.L., Kim, Y.S. (1989). Expression of Tn, sialosyl-Tn, and T antigens in human colon cancers. Cancer Res., 49, 197-204.

Itzkowitz, S.H., Bloom, E.J., Kokal, W.A., Modin, G., Hakomori, S., Kim, Y.S. (1990). Sialosyl-Tn: a novel mucin antigen associated with prognosis in colorectal cancer patients. Cancer, 66, 1960-66. 
Kobayashi, H., Terao, T., Kawashima, Y. (1991). Clinical evaluation of circulating serum sialyl Tn antigen levels in patients with epithelial ovarian cancer. J. Clin. Oncol., 9, 983-87.

Kobayashi, H., Terao, T., Kawashima, Y. (1992). Serum sialyl Tn as an independent predictor of poor prognosis in patients with epithelial ovarian cancer. J. Clin. Oncol., 10, 95-101.

Kurosaka, A., Kitagawa, H., Fukui, S., Numata, Y., Nakada, H., Funakoshi, I., Kawasaki, T., Ogawa, T., Iijima, H., Yamashina, I. (1988). A monoclonal antibody that recognises a cluster of a disaccharide, Neuo2-6GalNAc, in mucin-type glycoproteins. J. Biol. Chem., 263, 8724 26.

Layton, G.T., Stanworth, D.R., Amos, H.E. (1987). The specificity of murine polyclonal and monoclonal antibodies to the haptenic drug chlorhexidine induced by chlorine-generated chlorhexidine-protein conjugates. Clin. Exp. Immunol., 69, 157-65.

Loy, T.S., Nashelsky, M.B. (1993). Reactivity of B72.3 with adenocarcinomas. Cancer, 72, 2495-98.

Ma, X.C., Terata, N., Kodama, M., Jancic, S., Hosokawa, Y., Hattori, T. (1993). Expression of sialyl-Tn antigen is correlated with survival time of patients with gastric carcinomas. Eur. $J$. Cancer, 29A, 1820-23.

Maeda, K., Chung, Y.S., Onoda, N., Nakanishi, I., Nitta, A., Arimoto, Y., Yamada, N., Kondo, Y., Kato, Y., Sowa, M. (1994). Serum sialyl-Tn antigen level as a prognostic indicator in patients with gastric cancer. Int. J. Oncol., 4, 129-32.

Motoo, Y., Kawakami, H., Watanabe, H., Satomura, Y., Ohta, H., Okai, T., Makino, H., Toya, D., Sawabu, N. (1991). Serum sialyl-Tn antigen levels in patients with digestive cancers. Oncology, 48, 321-26.

Nance, K.V., Silverman, J.F. (1991). Immunocytochemical panel for the identification of malignant cells in serous effusions. Am. J. Clin. Pathol., 95, 867-74.

Ordonez, N.G. (1989). The immunohistochemical diagnosis of mesothelioma. Differentiation of mesothelioma and lung adenocarcinoma. Am. J. Surg. Pathol., 13, 276-91.

Ryuko, K., Iwanari, O., Nakayama, S., Iida, K., Kitao, M. (1992). Clinical evaluation of serum sialosyl-Tn antigen levels in comparison with CA125 levels in gynecologic cancers. Cancer, 69, 2368-78.

Ryuko, K., Iwanari, O., Kitao, M., Moriwaki, S. (1993). Immunohistochemical evaluation of sialylosyl-Tn antigens in various ovarian carcinomas. Gynecol. Oncol., 49, 215-24.

Sheibani, K., Esteban, J.M., Bailey, A., Battifora, H., Weiss, L.M. (1992). Immunopathologic and molecular studies as an aid to the diagnosis of malignant mesothelioma. Human Pathol., 23. 107-16.

Shield, P.W., Callan, J.J., Devine, P.L. (1994). Markers for metastatic adenocarcinoma in serous effusion specimens. Diagn. Cytopathol., 11, 237-45.

Takahashi, I., Maehara, Y., Kusumoto, T., Yoshida, M., Kakeji, Y., Kusomoto, H., Furusawa, M., Sugimachi,K. (1993). Predictive value of preoperative serum sialyl Tn antigen levels in prognosis of patients with gastric cancer. Cancer, 72, 1836-40.

Thornton, D.J., Devine, P.L., Hanski, C., Howard, M., Sheehan, J.K. (1994). Identification of two major mucins in respiratory secretions. Am. J. Resp. Crit. Care Med., 150, 823-32.

Tsuji, T., Osawa, T. (1986). Carbohydrate structures of bovine submaxillary mucin. Carbohyd. Res., 151, 391-402.

Yonezawa, S., Tachikawa, T., Shin, S., Sato, E. (1992). Sialosyl-Tn antigen. Its distribution in normal human tissues and its expression in adenocarcinomas. Am. J. Clin. Pathol., 98, 167-74. 


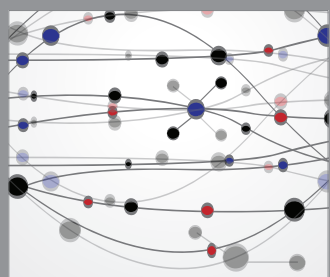

The Scientific World Journal
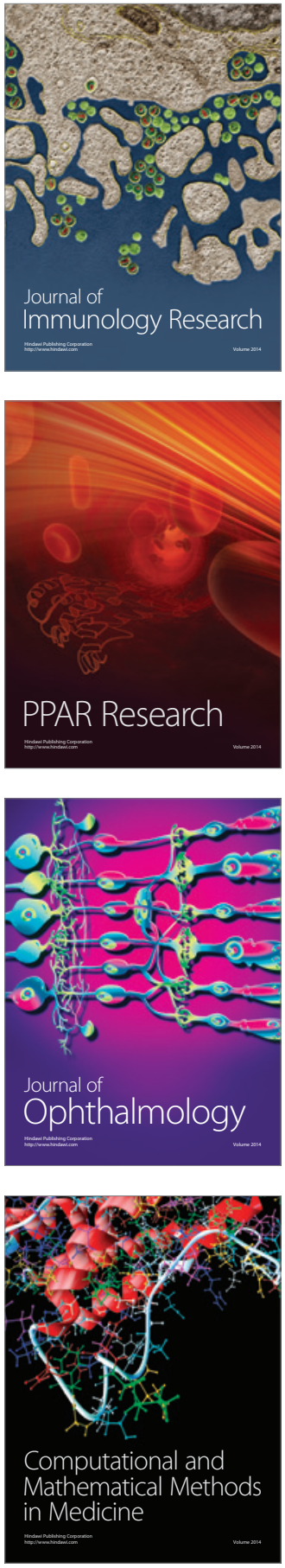

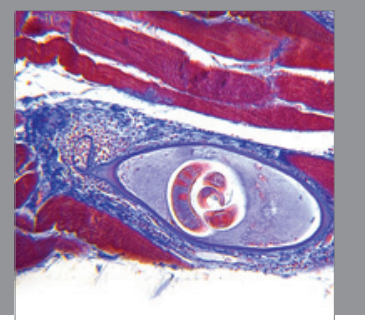

Gastroenterology

Research and Practice
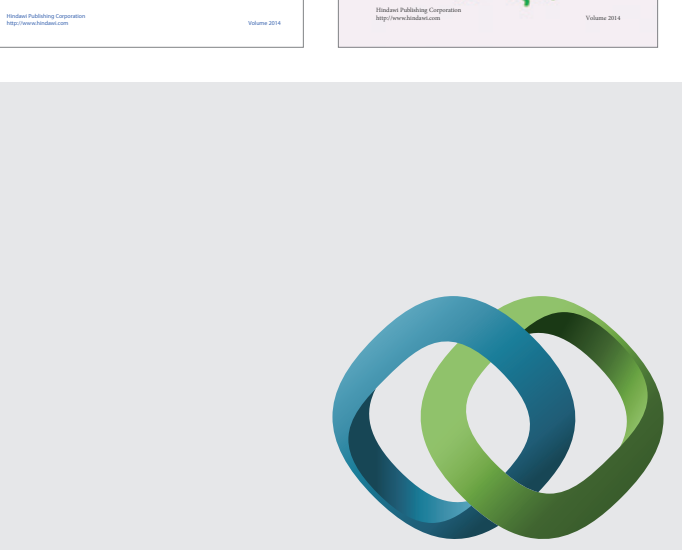

\section{Hindawi}

Submit your manuscripts at

http://www.hindawi.com
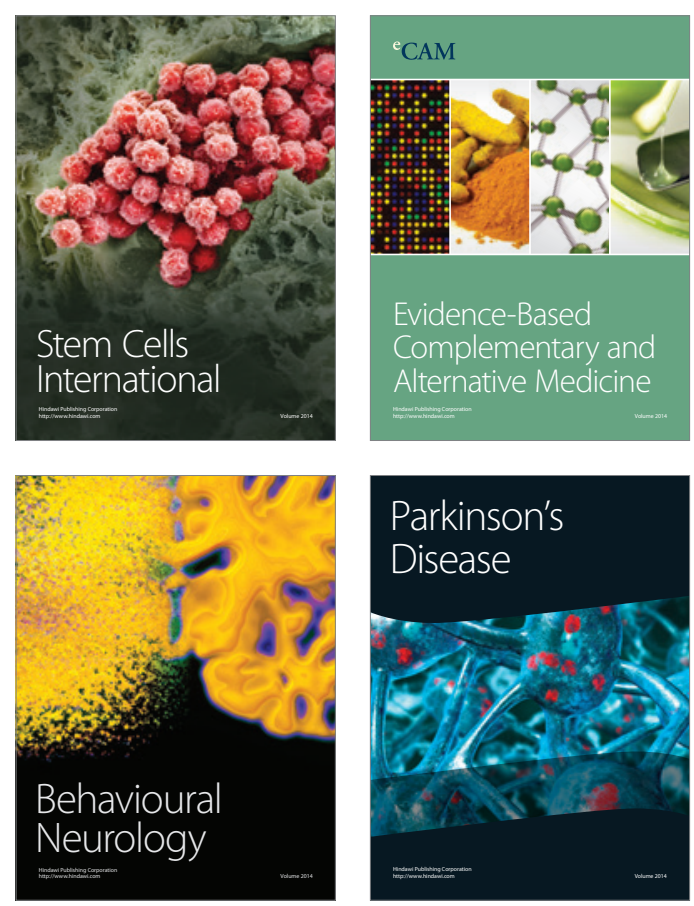

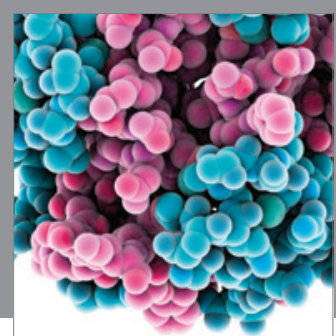

Journal of
Diabetes Research

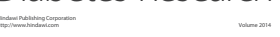

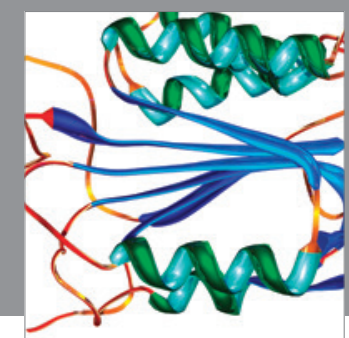

Disease Markers
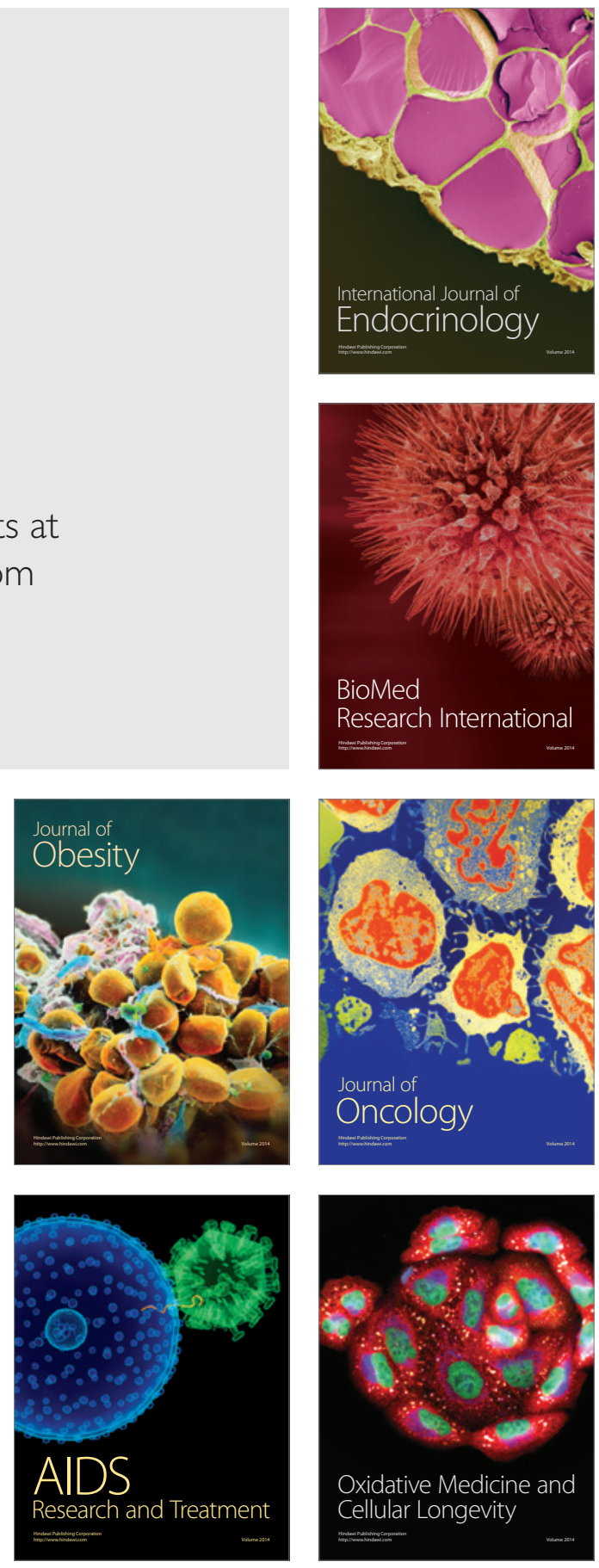